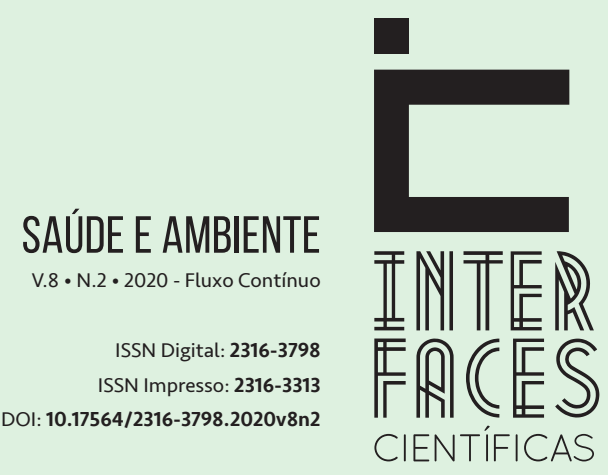

\section{ANÁLISE CLÍNICA E SALIVAR DAS CONDIÇÕES DE SAÚDE BUCAL DE UMACOMUNIDADE QUILOMBOLA DO NORDESTE BRASILEIRO}

\author{
CLINICAL AND SALIVARY ANALYSIS OF ORAL \\ HEALTH CONDITIONS OF A QUULOMBOLA COMMUNITY \\ IN THE NORTHEAST BRAZIL
}

\section{ANÁLISIS CLINIICO Y SALIVAR DE LAS CONDICIONES DE SALUD ORAL DE UNA QUULOMBOLA COMMUNITY ENEL NORESTE DE BRASIL}

Jamille Alves Araújo Rosa ${ }^{1}$ Matheus dos Santos Fernandez $z^{2}$ Cristiane Costa da Cunha Oliveira ${ }^{3}$

\section{RESUMO}

O objetivo deste estudo é analisar as condições de saúde bucal, fluxo e pH salivar, bem como o tipo de atendimento odontológico utilizado por uma população quilombola do Nordeste brasileiro. Participaram deste estudo um total de 86 pessoas, pertencentes às faixas etárias de 5 anos, 12 anos, 15 a 19 anos, 35 a 44 anos e 65 a 74 anos. A condição bucal em dentição permanente foi avaliada por meio do índice de dentes permanentes Cariados, Perdidos e Obturados (CPO-D), enquanto na dentição decídua utilizou-se o índice de dentes Cariados, com Extração indicada e Obturados (ceo-d). As variáveis sociodemográficas foram coletadas por meio de um questionário estruturado. A análise salivar ocorreu com o uso de um tubo coletor milimetrado e fita indicadora Macherey-Nagel. O processamento estatístico dos dados foi realizado no software SPSS 22.0, sendo que a análise descritiva das variáveis foi verificada por meio de frequência absoluta e média aritmética, e a análise bivariada adotou o Teste Exato de Fisher. Os resultados apontam elevados índices de ceo-d e CPO-D na comunidade. $\mathrm{Na}$ idade de 5 anos foi encontrado uma prevalência alta de cárie de $71,4 \%$ e na idade de 12 anos a média do CPO-D foi igual a 6,00. Nas faixas etárias de 15 a 19 anos; 35 a 44 e 65 a 74 as médias do CPO-D foram respectivamente: 6,$25 ; 15,38$ e 24,67 . Os fatores que estiveram associados à cárie dentária foram a idade $(p<0,001)$ e sexo $(p<0,001)$. Não foram encontradas alterações quanto ao $\mathrm{pH}$ e fluxo salivar. Concluiu-se que a prevalência de cárie dentária e edentulismo é alta na comunidade quilombola, refletindo a necessidade de criação e ampliação de políticas públicas de saúde voltadas para a melhoria da qualidade de vida dessa população. 


\section{PALAVRAS-CHAVE}

Epidemiologia; Inquéritos de Saúde Bucal; Saúde de grupos populacionais específicos; Cárie dentária

\section{ABSTRACT}

The aim of this study is to analyze oral health conditions, salivary flow and $\mathrm{pH}$, as well as the type of dental care used by a quilombola population in Northeast Brazil. An amount of 86 people participated in this study, belonging to the age groups of 5 years, 12 years, 15 to 19 years, 35 to 44 years and 65 to 74 years. We evaluated permanent dental condition by means of the index of permanent decayed, missing and filled teeth (DMFT), while in the primary dentition the decayed, missing and filled primary teeth ( $\mathrm{dmft}$ ) index. We collected sociodemographic variables using a structured questionnaire. Salivary analysis occurred with the use of a millimeter collection tube and a Macherey-Nagel indicator tape. The statistical processing of the data was performed using the SPSS 22.0 software, and variables descriptive analysis was verified by means of absolute frequency and arithmetic mean, and bivariate analysis adopted Fisher's exact test. The results indicate high rates of dmft and DMFT in the community, since at the age of 5 years a high prevalence of caries was found to be $71.4 \%$ and at the age of 12 the mean DMFT was equal to 6.00. In the age groups of 15 to 19 years; 35 to 44 and 65 to 74 means of the DMFT were, respectively: $6.25 ; 15.38$ and 24.67 . The factors that were associated with dental caries were age $(p<0.001)$ and sex $(p<0.001)$. There were no changes in $\mathrm{pH}$ and salivary flow. We concluded that the prevalence of dental caries and edentulism is high in quilombola community, reflecting the need to create and expand public health policies aimed at improving the quality of life of this population.

\section{KEYWORDS}

Epidemiology, Oral Health Surveys, Health of Specific Population Groups, Dental Caries

\section{RESUMEN}

El objetivo de este estudio es analizar las condiciones de salud bucal, el flujo salival y el pH, así como el tipo de cuidado dental utilizado por una población de quilombolas en el noreste de Brasil. Un total de 86 personas participaron en este estudio, pertenecientes a los grupos de edad de 5 años, 12 años, 15 a 19 años, 35 a 44 años y 65 a 74 años. La condición oral en la dentición permanente se evaluó mediante el índice de dientes permanentes cariados, faltantes y llenos (CPOD), mientras que en la dentición 
primaria el índice de dientes cariados, faltantes y llenos (ceo-d). Las variables sociodemográficas se recolectaron mediante un cuestionario estructurado. El análisis salival ocurrió con el uso de un tubo de recolección milimétrico y una cinta indicadora Macherey-Nagel. El procesamiento estadístico de los datos se realizó utilizando el software SPSS 22.0, y el análisis descriptivo de las variables se verificó mediante frecuencia absoluta y media aritmética, y el análisis bivariado adoptó la prueba exacta de Fisher. Los resultados indican altas tasas de dmft y DMFT en la comunidad, ya que a la edad de 5 años se encontró una alta prevalencia de caries del $71.4 \%$, e a la edad de 12 años la DMFT media fue igual a 6.00. En los grupos de edad de 15 a 19 años; 35 a 44 y 65 a 74 los medios de la DMFT fueron, respectivamente: $6.25 ; 15.38$ y 24.67. Los factores asociados con la caries dental fueron la edad $(p<0.001)$ y el sexo $(p<0.001)$. No hubo cambios en el $\mathrm{pH}$ y el flujo salival. Se concluyó que la prevalencia de caries dental y edentulismo es alta en la comunidad quilombola, lo que refleja la necesidad de crear y expandir políticas de salud pública destinadas a mejorar la calidad de vida de esta población.

\section{PALABRAS CLAVE}

Epidemiología; Encuestas de salud bucal; Salud de grupos de población específicos; Cavidad dental

\section{INTRODUÇ̄̃̃O}

Os problemas de saúde bucal têm sido cada vez mais abordados como importantes causadores de impacto negativo no desempenho diário e na qualidade de vida dos indivíduos (NÓBREGA et al., 2019). O relatório da Organização Mundial da Saúde (OMS) reconheceu que as doenças bucais causam dor, sofrimento, constrangimentos psicológicos e privações sociais, acarretando prejuízos em nível individual e coletivo (GOMES; ABEGG, 2007).

A desigualdade entre grupos étnicos quanto ao risco de cárie tem sido atribuída à pior condição socioeconômica de negros e pardos em relação aos brancos na sociedade brasileira, não sendo prevista a associação com outros fatores de ordem biológica (ANTUNES et al., 2006; ALVES et al., 2015). Gushi e colaboradores (2005) observaram que haviam índices mais elevados de cárie não tratada entre adolescentes pardos e negros, apontando piores condições de saúde para crianças não brancas.

Existem alguns trabalhos recentes sobre saúde bucal em comunidades remanescentes de quilombos, como o levantamento de Silva e colaboradores (2018), que apresentou resultados referentes ao acesso ao serviço odontológico e à prática de hábitos de higiene. Ademais, Rosa e colaboradores (2020) avaliaram a gravidade da condição periodontal e a ocorrência de microrganismos patogênicos na cavidade oral de uma população quilombola adulta no interior nordestino.

A cárie dentária continua sendo o principal problema de saúde bucal dos brasileiros. Entretanto, a situação melhorou de 2003 para 2010. Na idade de 12 anos, a doença atingia 69\% da população em 2003. Essa porcentagem diminuiu para 56\% em 2010. Esse declínio, de 13 pontos percentuais, corres- 
ponde a uma diminuição de $19 \%$ na prevalência da enfermidade. Os resultados da Pesquisa Nacional de Saúde Bucal (Projeto SB Brasil 2010) indicaram que, de acordo com o índice de dentes Cariados, Perdidos e Obturados (CPO-D), o Brasil saiu de uma condição de média prevalência de cárie em 2003 (CPO entre 2,7 e 4,4), para uma condição de baixa prevalência em 2010 (CPO entre 1,2 e 2,6) (BRASIL, 2012).

Os estudos epidemiológicos em saúde bucal na população brasileira também são escassos, principalmente no que se refere à zona rural quilombola (BIDINOTTO et al., 2017; RODRIGUES et al., 2011). 0 primeiro levantamento epidemiológico em saúde bucal delineado, considerando além das capitais, os interiores dos estados brasileiros foi o de 2010 (BRASIL, 2012). Entretanto, provavelmente os resultados referentes à saúde bucal em comunidades específicas como os quilombolas, não foram apresentados com foco necessário para permitir o planejamento adequado para essas populações.

Neste contexto, o objetivo deste trabalho foi analisar as condições de saúde bucal, pH e fluxo salivar e acesso ao atendimento odontológico de uma população quilombola do Nordeste brasileiro. A definição deste panorama epidemiológico, com características específicas nesta comunidade, facilitará o planejamento de ações de promoção e prevenção em saúde bucal.

\section{MÉTODO}

Este estudo, de caráter observacional e transversal, foi realizado no povoado Patioba, município de Japaratuba no Estado de Sergipe, situado no nordeste brasileiro. 0 espaço físico do povoado (Patioba 1 e 2) foi distribuído em diferentes ruas, nomeadas como A, B, C e D para facilitar o levantamento. Todas essas ruas foram igualmente selecionadas para a pesquisa. Essa distribuição foi usada como estratégia para identificar as residências da comunidade durante o desenvolvimento da pesquisa, visto que, não havia registro oficial dos logradouros existentes na comunidade.

Em 2009 a população desse povoado era composta de 593 habitantes, correspondendo a 186 famílias cadastradas no Sistema de Informação da Atenção Básica do SUS. Foram incluídos no estudo todos os indivíduos de 5 anos; 12 anos; 15 a 19 anos; 35 a 44 anos e 65 a 74 anos, de ambos os gêneros que concordaram e assinaram o Termo de Consentimento Livre e Esclarecido (TCLE), previamente apresentado. Esses grupos etários foram selecionados devido ao fato do levantamento seguir criteriosamente o protocolo de seleção etária do Levantamento Nacional de Saúde Bucal de 2010 para facilitar futuras comparações (BRASIL, 2012).

Em relação aos indivíduos com idade menor ou igual a 18 anos, o TCLE foi assinado pelo responsável legal. Foram excluídos os hospitalizados. Após essa seleção a amostra contabilizou a participação de 85 sujeitos, deles 18 eram crianças (5 e 12 anos), 32 eram adolescentes (15 a 19 anos), 29 eram adultos ( 35 a 44 anos) e 6 eram idosos ( 65 a 74 anos).

A análise sociodemográfica foi realizada por meio da aplicação de um questionário adaptado do Instituto Brasileiro de Geografia e Estatística (IBGE). Nas crianças de 5 anos e de 12 anos, esses questionários não foram preenchidos, sendo utilizada a ficha clínica, contendo algumas questões relacionadas ao acesso ao serviço odontológico, preenchidas pelo responsável legal. 
Foi realizado o exame clínico bucal para investigar as condições de cárie dentária na dentição decídua e permanente, por meio dos índices de dentes ceo-d e CPO-D, respectivamente. 0 índice de dentes Cariados, com Extração indicada e Obturados (ceo-d) inclui a contagem dos dentes decíduos cariados, com extração indicada e restaurados e varia de 0 a 20. Já o índice CPO-D é resultante do somatório dos dentes permanentes cariados, perdidos e restaurados e varia de 0 a 28. 0 edentulismo foi analisado nos grupos etários de 15 a 19 anos, 35 a 44 anos e 65 a 74 anos.

Os critérios de diagnóstico e a metodologia foram os da Pesquisa Nacional de Saúde Bucal - Projeto SB Brasil 2010 (BRASIL, 2012). A pesquisa foi realizada por examinadores previamente calibrados, utilizando as seguintes ferramentas recomendadas pela Organização Mundial da Saúde (WHO, 2013): odontoscópio, sonda exploradora número 5, pinça clínica, algodão, espátula de madeira e luvas de procedimento.

Adicionalmente, foi realizada análise da saliva quanto ao fluxo e $\mathrm{pH}$ em adolescentes, adultos e idosos. A saliva foi coletada em coletor de plástico milimetrado no intervalo de 1 a 3 minutos no turno matutino, após o dejejum, sem nenhum tipo de estimulação. Após o sujeito cuspir, eram gotejados de 4 a 5 gotas de dimeticona, agente antiespumante que permite induzir uma rápida precipitação de espuma salivar, mexendo vigorosamente com uma espátula de madeira para incorporá-lo à saliva. A taxa do fluxo salivar foi expressa em mililitros por minuto $(\mathrm{ml} / \mathrm{min})$.

Os valores foram analisados da seguinte forma: fluxo normal, de 1,0 a 3,0 ml/min; baixo fluxo, de 0,7 a $1,0 \mathrm{ml} / \mathrm{min}$; hipossalivação, menos de $0,7 \mathrm{ml} / \mathrm{min}$. Para a análise do $\mathrm{pH}$ salivar utilizou-se uma fita indicadora Macherey-Nagel com faixas de 0-14, mergulhada em saliva para quantificar o $\mathrm{pH}$ salivar e posteriormente comparou-se com a fita indicadora correspondente, conforme indicação do fabricante.

A análise estatística foi conduzida a partir do software SPSS 22.0, com cálculo das médias dos índices de cárie dentária. Para dentes permanentes foi realizado cálculo das médias dos índices globais de CPO-D e CEO-D, além da média de cada um dos seus componentes. Foi aplicado teste exato de Fisher para associação das médias de CPO-D com as variáveis sociodemográficas. Para realização das análises bivariadas, a variável CPO-D foi categorizada. Os resultados com $p$-valor $\leq 0,05$ foram considerados estatisticamente significantes. 0 estudo foi aprovado pelo Comitê de Ética em Pesquisa da Universidade Tiradentes, sob protocolo de número 270610.

\section{RESULTADOS}

Neste estudo foram examinados 86 indivíduos, de ambos os sexos, 7 na idade de 5 anos, 12 na idade de 12 anos, 32 na faixa etária de 15 a 19 anos, 29 na faixa etária de 35 a 44 anos e 6 na faixa etária de 65 a 74 anos para determinar a prevalência e severidade da cárie dentária. Os resultados apontam elevados índices de ceo-d e CPO-D na população quilombola. Na idade de 5 anos foi encontrado uma média de ceo-d de 5,71 e na idade de 12 anos a média do ceo-d foi de 6,00. No presente estudo a média do CPO-D na faixa etária de 15 a 19 anos foi de 6,25.

Além disso, O CPO-D médio da população apresentou valor elevado na faixa etária de 35 a 44 anos. Dos 29 indivíduos analisados, obteve-se média CPO-D 15,38. Entre os idosos, foram analisados 
6 indivíduos cuja média do CPO-D foi de 24,67. Este estudo apontou que na população adulta estudada no componente de dentes perdidos, a média obtida foi de 11,34 e para a população idosa esse componente apresentou uma média de 23 , ambas consideradas elevadas (TABELA 1).

Tabela 1 - Distribuição das médias de dentes hígidos $(\mathrm{H})$, cariados $(\mathrm{C})$, perdidos $(\mathrm{P})$, obturados (O), e índice ceo-d/CPO-D, por faixa etária da comunidade quilombola Patioba, Japaratuba, Sergipe, Brasil. (N=67)

\begin{tabular}{ccccccc}
\hline Idade (anos) & N & H & C & P | O & $\mathbf{0}$ & ceo-d| CPO-D \\
\hline 5 anos & 7 & 15,0 & 4,3 & 0,9 & 0,6 & 5,7 \\
12 anos & 12 & 19,6 & 4,7 & 0,3 & 1,0 & 6,0 \\
15 a 19 anos & 32 & 22,6 & 0,8 & 1,0 & 4,1 & 6,3 \\
35 a 44 anos & 29 & 15,7 & 1,1 & 11,3 & 2,9 & 15,4 \\
65 a 74 anos & 6 & 6,8 & 1,0 & 23,0 & 0,7 & 24,7 \\
\hline
\end{tabular}

Fonte: Dados da pesquisa.

A análise dos fatores sociodemográficos como sexo, idade, renda mensal, nível de escolaridade e acesso aos serviços de saúde com o CPO-D categorizado demonstrou que não houve correlação significativa entre a renda $(p=0,630)$, nível de escolaridade $(p=0,360)$ e acesso aos serviços de saúde $(p=0,691)$ com o CPO-D nas diferentes constantes. Apenas as variáveis sexo $(p<0,001)$ e idade $(p<0,001)$ apresentaram associação significativa com os grupos do CPO-D, onde os indivíduos do sexo masculino apresentaram-se com os maiores valores das constantes do CPO-D, distribuídos de 6 a 14 e mais que 21 elementos dentários afetados. Em relação à idade, aqueles indivíduos inclusos na faixa-etária de 34 a 44 anos apresentaram o valor de CPO-D entre os grupos de 10 a 20 dentes afetados (TABELA 2).

Tabela 2 - Análise das categorias do índice CPO-D de acordo com as características sociodemográficas e de acesso ao serviço de atendimento à saúde bucal na comunidade quilombola Patioba, Japaratuba, Sergipe, Brasil. $(\mathrm{N}=67)$

\begin{tabular}{|c|c|c|c|c|c|c|c|}
\hline \multirow{2}{*}{ Variáveis } & \multicolumn{6}{|c|}{$\begin{array}{c}\text { CPO-D } \\
\text { Categorias (n) }\end{array}$} & \multirow{2}{*}{ p-valor* } \\
\hline & 0 a 2 & 3 a 5 & 6 a 9 & 10 a 14 & 15 a 20 & $\geq 21$ & \\
\hline \multicolumn{8}{|l|}{ Sexo } \\
\hline Masculino & 4 & 3 & 10 & 13 & 8 & 10 & \multirow{3}{*}{$<0,001$} \\
\hline & & & & & & & \\
\hline Feminino & 5 & 4 & 5 & 2 & 2 & 1 & \\
\hline
\end{tabular}




\begin{tabular}{|c|c|c|c|c|c|c|c|}
\hline \multirow{2}{*}{ Variáveis } & \multicolumn{6}{|c|}{$\begin{array}{c}\text { CPO-D } \\
\text { Categorias (n) }\end{array}$} & \multirow[t]{2}{*}{ p-valor* } \\
\hline & 0 a 2 & 3 a 5 & 6 a 9 & 10 a 14 & 15 a 20 & $\geq 21$ & \\
\hline \multicolumn{8}{|l|}{ Idade } \\
\hline 15 a 19 anos & 8 & 6 & 11 & 6 & 1 & 0 & \multirow{3}{*}{$<0,001$} \\
\hline 35 a 44 anos & 1 & 1 & 3 & 9 & 9 & 6 & \\
\hline 65 a 74 anos & 0 & 0 & 1 & 0 & 0 & 5 & \\
\hline \multicolumn{8}{|l|}{ Renda } \\
\hline Sem renda fixa & 3 & 3 & 5 & 4 & 3 & 2 & \multirow{4}{*}{0,630} \\
\hline Até um SM & 1 & 2 & 4 & 5 & 1 & 5 & \\
\hline Até $2 \mathrm{SM}$ & 4 & 1 & 3 & 6 & 5 & 2 & \\
\hline Até 3 SM & 1 & 0 & 0 & 0 & 0 & 0 & \\
\hline \multicolumn{8}{|l|}{ Nivel de escolaridade } \\
\hline Educação básica & 1 & 1 & 1 & 2 & 1 & 7 & \multirow{5}{*}{0,360} \\
\hline Ensino Fundamental incompleto & 3 & 3 & 3 & 8 & 1 & 3 & \\
\hline Ensino Fundamental completo & 0 & 0 & 1 & 3 & 0 & 0 & \\
\hline Ensino médio incompleto & 4 & 3 & 6 & 2 & 2 & 0 & \\
\hline Ensino médio completo & 1 & 0 & 4 & 0 & 6 & 1 & \\
\hline \multicolumn{8}{|l|}{ Acesso ao serviço de Saúde } \\
\hline Particular & 1 & 0 & 2 & 1 & 1 & 1 & \multirow{4}{*}{0,691} \\
\hline Público & 6 & 4 & 10 & 9 & 5 & 9 & \\
\hline Convênio pela empresa & 0 & 3 & 3 & 5 & 4 & 1 & \\
\hline $\begin{array}{l}\quad \text { Convênio pessoal } \\
\text { SM - Salário Mínimo } \\
\text { * Teste exato de Fisher }(p<0.005) \\
\text { Fonte: Dados da pesquisa. }\end{array}$ & 1 & 0 & 0 & 0 & 0 & 0 & \\
\hline
\end{tabular}

Importantes fatores biológicos como o pH e fluxo salivar também foram analisados em adolescentes, adultos e idosos. A análise descritiva do fluxo e $\mathrm{pH}$ salivar demonstrou que os indivíduos entre 15 e 44 apresentaram pH e fluxo salivar dentro dos padrões de normalidade (6,8 a 7,2). 0 valor de $\mathrm{pH}$ médio dos indivíduos analisados foi de 6,76 e apenas $22 \%$ dos sujeitos apresentaram baixo fluxo salivar ou hipossalivação (TABELA 3). 
Tabela 3 - Fluxo e pH salivar dos indivíduos adolescentes, adultos e idosos da comunidade quilombola Patioba, Japaratuba, Sergipe, Brasil. $(\mathrm{N}=67)$

\begin{tabular}{ccccccccc}
\hline & \multicolumn{3}{c}{ Fluxo salivar } & \multicolumn{3}{c}{ pH } \\
\cline { 2 - 8 } Faixa etária & $\mathbf{N}$ & Normal & Baixo fluxo & Hipossa-livação & $\begin{array}{l}\text { Desvio } \\
\text { padrão }\end{array}$ & min & max \\
\hline 15 a 19 anos & 32 & 27 & 2 & 3 & 0,672 & 6 & 8 \\
35 a 44 anos & 29 & 23 & 3 & 3 & 0,577 & 5 & 8 \\
65 a 74 anos & 6 & 2 & 3 & 1 & 0,548 & 6 & 7 \\
\hline
\end{tabular}

Fonte: Dados da pesquisa.

Em relação a utilização de serviços odontológicos, a maioria da população estudada faz uso do serviço público de saúde (78\%), principalmente crianças, adolescentes e idosos. Os adultos utilizam, além do atendimento público, outros convênios empresariais (20\%) e serviço particular (6\%) (TABELA 4).

Tabela 4 - Tipo de serviço odontológico utilizado pelos participantes da pesquisa por idade na população quilombola Patioba, Japaratuba, Sergipe, Brasil. (N=86)

\begin{tabular}{cccccc}
\hline Idade (anos) & N & Particular & Público & Convênio Empresarial & Convênio Pessoal \\
\hline 5 anos & 7 & 0 & 7 & 0 & 0 \\
12 anos & 12 & 0 & 11 & 1 & 0 \\
15 a 19 anos & 32 & 3 & 27 & 7 & 1 \\
35 a 44 anos & 29 & 2 & 17 & 10 & 0 \\
65 a 74 anos & 6 & 1 & 5 & 0 & 0 \\
\hline
\end{tabular}

Fonte: Dados da pesquisa.

\section{DISCUSSÃO}

Os resultados apontam elevados índices de ceo-d e CPO-D na população. Na idade de 5 anos foi encontrado uma média de ceo-d de 5,71 e na idade de 12 anos a média do ceo-d foi de 6,25 (TABELA 1). No Levantamento Nacional de Saúde Bucal Brasileira de 2010, a média do índice do CPO-D aos 12 anos ficou em 2,1, correspondendo à redução de $25 \%$ quando comparada à média do levantamento de 2003 (BRASIL, 2012). 
Outros autores descreveram os diferenciais urbano-rurais da prevalência de cárie dentária em crianças com dentição decídua no Estado de São Paulo e identificaram fatores associados. Participaram do estudo 24.744 crianças de 5 a 7 anos examinadas no Levantamento Epidemiológico de Saúde Bucal do Estado de São Paulo. A classificação por cor da pele, definida pelo cirurgião-dentista que efetuou o exame bucal, foi estudada em duas categorias: negros (crianças de descendência africana, incluindo as que foram classificadas como pardas) e não negros (crianças brancas de descendência europeia). Apresentaram maior prevalência de cárie as crianças negras e pardas (em relação às brancas) e as crianças matriculadas em escolas públicas (em relação às matriculadas em escolas privadas), tanto em áreas urbanas como rurais (MELLO et al., 2008).

No SB Brasil 2010, entre os adolescentes de 15 a 19 anos, a média de dentes afetados foi de 4,2, exatamente o dobro do número médio encontrado aos 12 anos. Esta evolução do CPO entre 12 e 15-19 anos tem sido um achado comum em outros estudos no Brasil e no mundo (OMS, 2003). Comparando-se com 2003, contudo, a redução no componente cariado foi de $39 \%$ (de 2,8 dentes em 2003, para 1,7 em 2010) (BRASIL, 2012). No presente estudo a média do CPO-D na faixa etária de 15 a 19 anos foi de 6,25, sendo esta uma média superior comparada aos resultados do SB Brasil 2010.

Em um estudo realizado por Gushi e colaboradores (2005), foi analisada a cárie dentária em 1.825 adolescentes de 15 a 19 anos de idade no Estado de São Paulo. Os dados obtidos foram estratificados segundo a idade, gênero, etnia e municípios com e sem fluoretação das águas de abastecimento público. Considerando a variável etnia, as diferenças entre as médias CPO-D não foram estatisticamente significantes na amostra total. Entretanto, existiram diferenças com relação aos componentes do índice CPO-D: o grupo dos não brancos teve maior porcentagem de dentes cariados $(p<0,001)$ e perdidos $(p<0,001)$, enquanto os brancos obtiveram maior porcentagem de dentes obturados $(p<0,001)$.

0 presente estudo analisou uma população adulta afrodescendente com média CPO-D elevado na faixa etária de 35 a 44 anos. Dos 29 indivíduos analisados obteve-se média CPO-D 15,38. Entre os idosos, foram analisados 6 indivíduos cuja média do CPO-D foi de 24,6. Estes resultados acompanharam a tendência brasileira do último levantamento cujo CPO-D médio na faixa etária de 35 a 44 anos foi de 16,3 com redução de 19\% em relação ao levantamento anterior em 2010 (BRASIL, 2012). Os resultados para a população idosa foram similares ao último levantamento epidemiológico brasileiro cuja média foi de 27,1 (BRASIL, 2012).

Entre os componentes do CPO-D analisados no levantamento da população adulta, o componente de dentes perdidos apresentou a média mais elevada, 11,34 e para a população idosa, esse componente apresentou uma média de 23, ambas consideradas elevadas. Esse achado foi semelhante a estudos brasileiros como o de Antunes e colaboradores (2006), que encontrou uma média do componente perdido de 11,57 para população adulta de 35 a 44 anos de professores e funcionários de escolas públicas e particulares do estado de São Paulo. No estudo foi observada a ocorrência de taxas crescentes de perda dentária precoce em adultos mais velhos, negros, que trabalhavam em escolas rurais, em cidades pequenas e que residiam em áreas de água de abastecimento não fluoretada.

A desigualdade entre grupos étnicos quanto ao risco de cárie tem sido atribuída à pior condição socioeconômica de negros e pardos em relação aos brancos na sociedade brasileira, não estando prevista 
a associação de outros fatores de ordem biológica (ANTUNES et al., 2006). Pode-se observar o elevado valor da média do componente perdido para a população adulta e idosa, no presente estudo foi elevada e corresponde a presença de edentulismo. 0 componente de dentes perdidos aumentou com a idade. Esse resultado deve ser um indicador para os gestores públicos de saúde para que políticas de promoção de saúde e acesso ao tratamento restaurador sejam planejadas para todas as faixas etárias.

Importantes fatores biológicos, como o pH e fluxo salivar, também foram analisados neste estudo. A frequência descritiva do $\mathrm{pH}$ salivar demonstrou que a maioria dos indivíduos pesquisados apresentou valor médio de 6,75 e fluxo salivar dentro dos padrões de normalidade, com menos da metade dos sujeitos em condições de hipossalia ou baixo fluxo salivar.

A mensuração do índice de $\mathrm{pH}$ salivar interfere no conhecimento da qualidade da saliva, visto que quanto menor os valores de pH, maior é a propensão do risco de lesão cariosa (CUNHA-CRUZ et al., 2013). Além disso, O fluxo salivar normal também garante as condições de saúde bucal, evitando doenças como cárie, periodontopatias, halitose e desconforto do paciente (ardência, perda do paladar, dificuldade de fonação, mastigação, deglutição e o uso de prótese), observadas quando há redução do fluxo salivar (COUTO; LOPES, 2010).

A literatura científica demonstra que ainda existem discrepâncias na Atenção à Saúde Bucal e no acesso às políticas de saúde dos povoados quilombolas brasileiras, sugerindo que a reversão desse quadro somente ocorrerá com um maior comprometimento de seus profissionais e investimentos em infraestrutura na saúde pública (FREITAS et al., 2011; BEZERRA et al., 2014).

No presente estudo a maioria da população estudada faz uso do serviço público de saúde, principalmente crianças, adolescentes e idosos. Já os adultos utilizam, além do convênio público, outros convênios empresariais e serviço privado. Embora a população apresente elevados valores para os índices ceo-d e CPO-D, parece existir um acesso adequado ao serviço público para a população infantil e adolescentes. Esse fato pode ser justificado pela carência da atenção primária e acesso a informações na localidade.

A política governamental brasileira para a saúde bucal compreende que se deve ampliar e qualificar o acesso ao atendimento básico, garantindo serviços odontológicos em todas as unidades básicas de saúde, incluindo áreas rurais, de difícil acesso e de fronteiras nacionais, com atendimentos em horários que possibilitem o acesso das pessoas, com ênfase para a implementação de ações de saúde bucal junto aos remanescentes de quilombolas (BRASIL, 2005). De acordo com a Portaria no 1.434, de 14/7/2004, conhecida como de incentivo à equidade, que, dentre outras providências e fixando critérios específicos, aumenta em $50 \%$ o valor atual do repasse relativo às equipes de Saúde da Família e Saúde Bucal que, nos municípios com população remanescente de quilombos, atuam junto as comunidades (BRASIL, 2004).

0 relatório da $3^{a}$ Conferência em Saúde Bucal colocou entre as prioridades implementar ações de saúde bucal junto às populações indígenas e remanescentes de quilombos, após ampla discussão com as suas organizações, a fim de se garantir o estabelecimento de um programa de atendimento de caráter não-mutilador, universal, integral e com equidade, que considere as experiências e os valores culturais relacionados às práticas higiênicas e dietéticas de cada povo indígena ou quilombola (PUCCA JR, 2006).

A literatura aponta a ênfase que tem sido dada a importância de implementar ações de saúde bucal junto às populações remanescentes de quilombos, após ampla discussão com as suas orga- 
nizações, a fim de se garantir o estabelecimento de um programa de atendimento de caráter não-mutilador, universal, integral e com equidade, que considere as experiências e os valores culturais relacionados às práticas higiênicas e dietéticas de cada povo quilombola (SANTOS; MAIO, 2004; NARVAl, 2006; PARÉ et al., 2007; MACHADO et al., 2010).

É fundamental que todas as profissões de saúde despertem para este grande desafio e possam se unir numa corrente inter, multi e transdisciplinar, aumentando a proximidade a importantes grupos de brasileiros que, por uma motivação triste de nosso passado, estiveram e ainda estão excluídos do processo atual de desenvolvimento (FREITAS et al., 2011).

No presente estudo, devido ao seu caráter transversal, número amostral exíguo e o fato de apenas uma comunidade quilombola ter sido incluída possui fatores limitantes para estabelecer inferências causais e generalização da população. Todavia, este estudo evidenciou informações importantes quanto as condições de saúde bucal da comunidade quilombola Patioba, principalmente pelas características específicas da própria comunidade.

\section{CONCLUSÃO}

De maneira geral, foi verificada alta prevalência de cárie dentária entre todas as faixas-etárias avaliadas no estudo, principalmente entre os sujeitos do sexo masculino, bem como alto índice de edentulismo entre os indivíduos mais velhos. Não foram observadas alterações da normalidade nas investigações das características salivares. Em relação ao tipo de serviço de atenção em saúde bucal frequentemente utilizado pela comunidade, a grande maioria busca atendimento no serviço público.

Esses resultados demonstram que nessa comunidade existe uma necessidade de maior enfoque e desenvolvimento de ações no campo da saúde bucal, tanto na promoção e cuidados para crianças e adolescentes, mas, principalmente, com atenção específica para prevenção e reabilitação da população adulta e idosa.

\section{REFERÊNCIAS}

ALVES, N. S. et al. Determinantes sociais e sua relação com a experiência de cárie em adolescentes: um estudo transversal. J. Health. Sci., v. 17, n. 2, p. 93-99, 2015.

ANTUNES, J. L. F. et al. Multilevel assessment of determinants of dental caries experience in Brazil. Community Dent. Oral, v. 34, n. 2, p. 146-152, 2006.

BEZERRA, V. M. et al. Inquérito de saúde em comunidades quilombolas de Vitória da Conquista, Bahia, Brasil (Projeto COMQUISTA): aspectos metodológicos e análise descritiva. Cien. Saude Col., v. 19, p. 1835-1847, 2014. 
BIDINOTTO, A. B. et al. Autopercepção de saúde bucal em comunidades quilombolas no Rio Grande do Sul: um estudo transversal exploratório. Rev. Bras. Epidemiol., v. 20, p. 91-101, 2017.

BRASIL. Ministério da Saúde. Portaria n 1.434/GM. Mudanças no financiamento da atenção básica em saúde no âmbito da estratégia Saúde da Família. Brasília: Ministério da Saúde, 2004

BRASIL. Ministério da Saúde. Secretaria Especial de Políticas de Promoção da Igualdade Racial.

Programa Brasil Quilombola. Brasília: Ministério da Saúde, 2005.

BRASIL. Ministério da Saúde. Secretaria de Atenção à Saúde. Secretaria de Vigilância em Saúde. SB Brasil 2010: Pesquisa Nacional de Saúde Bucal - resultados principais. Brasília: Ministério da Saúde, 2012.

COUTO, J. A. D. M.; LOPES, F. F. The influence of age in the speed of salivary flow in adults. RFO UPF, v. 15, n. 2, p. 135-138, 2010.

CUNHA-CRUZ, J. et al. Salivary characteristics and dental caries: evidence from general dental practices. J. Am. Dent. Assoc., v. 144, n. 5, p. 31-40, 2013.

FREITAS, D. A. et al. Saúde e comunidades quilombolas: uma revisão da literatura. Rev. CEFAC, v. 13, n. 5, p. 937-943, 2011.

GOMES, A. S.; ABEGG, C. O impacto odontológico no desempenho diário dos trabalhadores do Departamento Municipal de Limpeza Urbana de Porto Alegre, Rio Grande do Sul, Brasil. Cad. Saúde Publ., v. 23, p. 1707-1714, 2007.

GUSHI, L. L. et al. Cárie dentária em adolescentes de 15 a 19 anos de idade no Estado de São Paulo, Brasil, 2002. Cad. Saúde Publ., v. 21, p. 1383-1391, 2005.

MACHADO, C. V. et al. O planejamento nacional da política de saúde no Brasil: estratégias e instrumentos nos anos 2000. Cien. Saude Col., v. 15, p. 2367-2382, 2010.

MELLO, T. R. D. C. et al. Prevalência de cárie não tratada na dentição decídua em áreas urbanas e rurais do Estado de São Paulo, Brasil. Rev. Panam. Salud Publ., v. 23, p. 78-84, 2008.

NARVAI, P. C. Saúde bucal coletiva: caminhos da odontologia sanitária à bucalidade. Rev. Saúde Publ., v. 40, p. 141-147, 2006.

NÓBREGA, A. V. D. et al. Impacto da cárie dentária na qualidade de vida de pré-escolares mensurado pelo questionário PedsQL. Cien. Saude Col., v. 24, p. 4031-4042, 2019. 
PARÉ, M. L. et al. A educação para quilombolas: experiências de São Miguel dos Pretos em Restinga Seca (RS) e da Comunidade Kalunga de Engenho II (GO). Cad. CEDES, v. 27, n. 72, p. 215-232, 2007.

PUCCA JR, G. A. A política nacional de saúde bucal como demanda social. Cien. Saude Col., v. 11, p. 243-246, 2006.

RODRIGUES, S. A. et al. Educação em saúde em comunidades quilombolas. Rev. Gaúch. Odontol., v. 59, n. 3, p. 445-451, 2011.

ROSA, J. A. A. et al. Detection of oral Entamoeba gingivalis and Trichomonas tenax in adult quilombola population with periodontal disease. Odovtos - Int. J. Dent. Sci., v. 22, n. 2, p. 331-338, 2020.

SANTOS, R. V.; MAIO, M. C. Qual "retrato do Brasil”? Raça, biologia, identidades e política na era da genômica. MANA, v. 10, n. 1, p. 61-95, 2004.

SILVA, E. K. P. D. et al. Saúde bucal de adolescentes rurais quilombolas e não quilombolas: um estudo dos hábitos de higiene e fatores associados. Cien. Saude Col., v. 23, p. 2963-2978, 2018.

WHO - World Health Organization. Oral health surveys: basic methods. World Health Organization: Geneve, 2013.

WHO - World Health Organization. The World Oral Health Report, 2003. Continuous improvement of oral health in the 21st century - the approach of the WHO Global Oral Health Program. World Health Organization: Geneve, 2003. 
1 Cirurgiã-dentista; Doutoranda do Programa de PósGraduação em Saúde e Ambiente da Universidade Tiradentes - UNIT. E-mail: jamillealves@hotmail.com

2 Faculdade de Odontologia; Universidade Federal de Pelotas - Pelotas, Rio Grande do Sul.

E-mail: mathsantos.f@gmail.com

3 Cirurgiã-dentista; Professora titular do Programa de Mestrado em Saúde e Ambiente da Universidade Tiradentes - UNIT; Pesquisadora do Instituto de Pesquisa e Tecnologia. E-mail: criscunhaoliva@yahoo.com.br

\section{(). (1) (-)}

Este artigo é licenciado na modalidade acesso abertosob a Atribuição-Compartilhalgual CC BY-SA

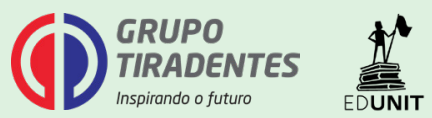

